\title{
Groups of galaxies in the SDSS Data Release 7
}

\section{Flux- and volume-limited samples ${ }^{\star}$}

\author{
E. Tago ${ }^{1}$, E. Saar ${ }^{1}$, E. Tempel ${ }^{1,3}$, J. Einasto ${ }^{1}$, M. Einasto ${ }^{1}$, P. Nurmi $^{2}$, and P. Heinämäki ${ }^{2}$ \\ 1 Tartu Observatory, 61602 Tõravere, Estonia \\ e-mail: erik@aai.ee \\ 2 Tuorla Observatory, Turku University, Väisäläntie 20, Piikkiö, Finland \\ 3 Institute of Physics, Tartu University, Tähe 4, 51010 Tartu, Estonia
}

Received 17 November 2009 / Accepted 19 February 2010

ABSTRACT

\begin{abstract}
Aims. We extract groups of galaxies as flux-limited and volume-limited samples from the SDSS Data Release 7 (DR7) to study the supercluster-void network and environmental properties of groups therein. Volume-limited catalogues are particularly useful for a comparison of numerical simulations of dark matter halos and the large-scale structure with observations.

Methods. The extraction of a volume-limited sample of galaxies and groups requires special care to avoid excluding too much observational data. We use a modified friends-of-friends ( FoF) method with a slightly variable linking length to obtain a preliminary flux-limited sample. We take the flux-limited groups as the basic sample to include as many galaxies as possible in the volume-limited samples. To determine the scaling of the linking length we calibrated group sizes and mean galaxy number densities within groups by magnitude dilution of a nearby group sub-sample to follow the properties of groups with higher luminosity limits.

Results. Our final flux-limited sample contains 78800 groups and volume-limited subsamples with absolute magnitude limits $M_{r}=$ $-18,-19,-20$, and -21 contain 5463, 12590,18973 , and 9139 groups, respectively, in the DR7 main galaxy main area survey. The group catalogue is available at the CDS.

Conclusions. The spatial number densities of our groups within the subsamples, as well as the mean sizes and rms velocities of our groups practically do not change from sub-sample to sub-sample. This means that the catalogues are homogeneous and well suited for a comparison with simulations.
\end{abstract}

Key words. cosmology: observations - large-scale structure of Universe - galaxies: clusters: general - catalogs

\section{Introduction}

Since the early days of extragalactic astronomy a well-known analogy between islands and galaxies is used; galaxies were frequently called "island universes". Now we face the fact that we rather live in an archipelago Universe: there are no really single or lonely galaxies. Groups and clusters of galaxies are natural environments for galaxies, and their study is important for understanding the evolution of galaxies, the dark matter problem, the large scale structure, and the underlying cosmological model.

The first extensive cluster catalogues by Abell (1958) and Abell et al. (1989) were created by visual inspection of the Palomar photographic plates. The next generation of galaxy group catalogues started from the Las Campanas catalogue of groups by Tucker et al. (2000) and was followed by the catalogues based on the 2 degree Field Galaxy Redshift Survey (2dFGRS) data releases (Colless et al. 2001, 2003) and the Sloan Digital Sky Survey (SDSS) data releases (Early Data Release, Data Release 1-Data Release 7) (Adelman-McCarthy et al. 2008). As one of several important results more search algorithms were developed (De Propris et al. 2002; Goto et al. 2002; Merchán \& Zandivarez 2002; Bahcall et al. 2003; Lee et al. 2004; Eke et al. 2004; Merchán \& Zandivarez 2005; Yang et al. 2005; Einasto et al. 2005; Weinmann et al. 2006;

* Full Table 2 is only available in electronic form at the CDS via anonymous ftp to cdsarc.u-strasbg.fr (130.79.128.5) or via http: //cdsweb.u-strasbg.fr/cgi-bin/qcat? J/A+A/514/A102
Tago et al. 2006; Berlind et al. 2006; Deng et al. 2007; Yang et al. 2007; Tago et al. 2008).

In our previous papers (Tago et al. 2006, 2008, hereafter Papers I and II) we extracted 2dFGRS and SDSS DR5 groups as flux-limited samples. In those papers we gave an extensive review of previous studies dedicated to group search methods, and of the published group catalogues. In this introduction we present a short review of studies of galaxy groups, and pay more attention to volume-limited samples.

Although a number of new group-finding algorithms have been developed (Goto et al. 2002; Kim et al. 2002; Bahcall et al. 2003; Nichol 2004; Koester et al. 2007; Yoon et al. 2008; Dong et al. 2008; Wen et al. 2009), the FoF method remains the most frequently applied for redshift surveys.

In recent years, several authors have compiled group catalogues using the 2dFGRS (Eke et al. 2004; Yang et al. 2005; Tago et al. 2006). Using earlier releases of the SDSS, preliminary catalogues of groups have been obtained (Einasto et al. 2003a; Lee et al. 2004; Merchán \& Zandivarez 2005; Goto 2005; Yang et al. 2005; Weinmann et al. 2006; Zandivarez et al. 2006; Berlind et al. 2006; Yang et al. 2007). However, similar algorithms used to compile these catalogues yielded groups of galaxies with rather different statistical properties. Several authors have presented catalogues of rich clusters of galaxies (Miller et al. 2005; Aguerri et al. 2007; Popesso et al. 2007) using the SDSS data. All these group and cluster catalogues are constructed on the basis of spectroscopic data, using certain 
selection criteria. We shall discuss and compare these catalogues with each other in a separate paper.

The most important problem in obtaining the catalogues of systems of galaxies has been the inhomogeneity of the resulting samples due to selection effects in search procedures. One of the ways to improve group search results is to use volumelimited samples instead of (or in addition to) flux-limited ones. For example, volume-limited samples are necessary for a comparison with $N$-body simulations and useful for testing subgroup extraction from groups and clusters of galaxies. In contrast to the majority of authors Berlind et al. (2006), indeed, has used volume-limited samples of the SDSS. This resulted in one of the most detailed search methods and reliable group catalogues.

The papers dedicated to group and cluster searches use a wide range of both sample selection methods as well as cluster search methods and parameters. The choice of the methods and parameters depends on the goal of the catalogue. In Papers I and II we also drew a conclusion that in previous group catalogues the luminosity-density relation in groups had not been taken into account.

In the present paper our goal is to generate a catalogue of groups comprised of the final public release (DR7) of the SDSS. We have applied the well-known FoF group search method to search for groups. To create a group catalogue we have taken into account the luminosity-density relation of galaxies in groups. As a final result we have extracted volume-limited galaxy samples and have applied a modified FoF group-finder method to obtain flux-limited group samples. As selection effects in data are important factors in choosing the galaxy groups, we have taken in the present paper into account selection effects as described in detail in Papers I and II.

The data used are described in Sect. 2. Section 3 discusses the group-finding algorithm. Selection effects that influence the choice of parameters for the FoF procedure are discussed in Sect. 4. To select an appropriate cluster-finding algorithm we analyse in Sect. 5 how the properties of groups change, if they are observed at various distances. We discuss the difficult problem how to define a correct volume-limited sample of groups of galaxies in Sect. 6. Section 7 describes step by step the procedures employed to select the groups. The catalogue itself is described in Sect. 8. In Sect. 9 we describe the main properties of the groups. In Sect. 10 we discuss a few important open issues and present our conclusions. As in Papers I and II we use for simplicity the term "group" for all objects in our catalogue, including clusters of galaxies.

\section{Data}

In this paper we have used the seventh, final data release of the SDSS (Adelman-McCarthy et al. 2006, 2007, 2008; Abazajian et al. 2009) that contains galaxies with observed spectra.

We have restricted our study to the main galaxy sample obtained from the SDSS Data Archive Server (DAS of March 2009), with 697920 galaxies. This sample was selected as the standard main galaxy sample described by Adelman-McCarthy et al. (2008) and the SDSS web site. The survey consists of a main contiguous area (hereafter the main area), three narrow strips in the southern sky, and a short strip at a high declination. We have excluded the smaller areas from our group search and concentrated on the main area, limited by the SDSS coordinate ranges $-75<\lambda<+75$ and $-40<\eta<+45$ (see Fig. 1).

We applied a lower redshift limit $z=0.009$ to our sample, to exclude galaxies of the Local Supercluster. As the SDSS sample

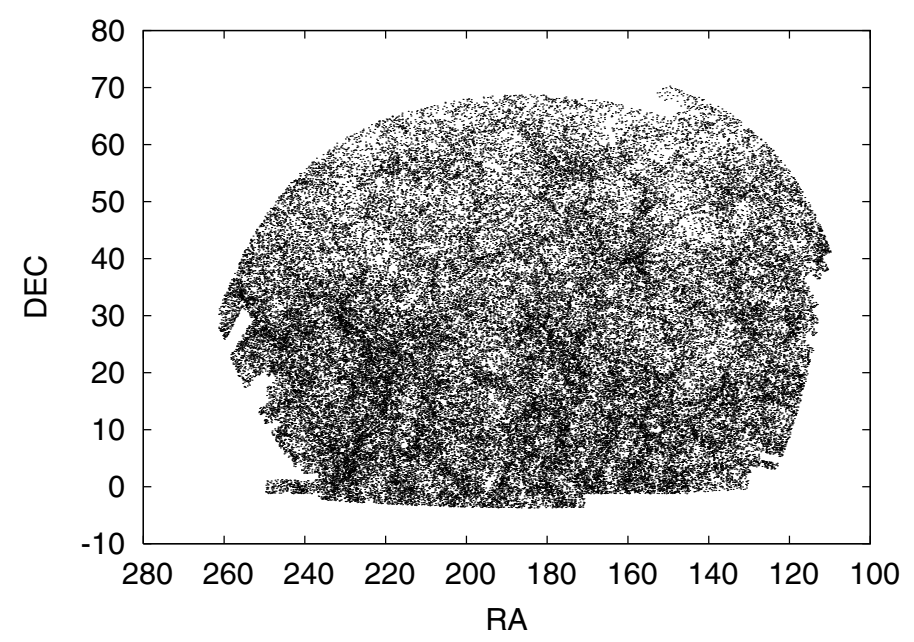

Fig. 1. Groups of galaxies in the flux-limited main area sample of the SDSS DR7 in equatorial coordinates. The distribution of groups is not uniform because the sample occupies a relatively thin shell and incompleteness of the magnitude-limited sample. Also some nearby structures might be followed.

becomes very diluted at large distances, we restricted our sample by a upper redshift limit $z=0.2$.

The spectroscopic survey is complete up to the Petrosian magnitude $r=17.77$ (Adelman-McCarthy et al. 2007). There is a serious problem of incompleteness at the bright end of galaxy magnitudes, due to saturation of bright galaxies and due to blending by saturated stars. However, as one of our purposes was to obtain nearby volume-limited samples we did not severely restrict the initial galaxy sample at the bright end. After correction of magnitudes for the galactic extinction we restricted our survey to galaxies of the Petrosian magnitudes from $r=12.5$ to $r=17.77$. Another important reason for incompleteness are the fiber collisions due to the minimum separation of $55^{\prime \prime}$ between spectroscopic fibers. For this reason about $6 \%$ of galaxies in the SDSS are without observed spectra. We discussed in detail the problem of poor de-blending in nearby groups and carried out visual inspection of 139 groups (Paper II, Sect. 8.1). As a result of the visual inspection we have these conclusions: a) bad de-blending has only a minor effect on our large sample of groups; b) comparison of these visually inspected groups with the same(duplicate) well-defined groups obtained by other authors gives us an additional test that our groupfinding algorithm does provide real groups and helps to find empirically initial linking lengths at $z=0$.

The biases mentioned above result in incompleteness of our group samples. It also affects the distribution of group properties. Unfortunately, there is no sufficient information to account for these incompleteness factors. Our main purpose in creating the SDSS group catalogues was to describe the large scale distribution of galaxies and their systems, and to obtain the luminosity density field in the nearby volume. This purpose does not need accurate determination of the properties of every single group. Therefore, we may hope that the biases discussed above do not affect the overall large-scale distribution of luminous matter, obtained from our group catalogue.

We found duplicates due to repeated spectroscopy for a number of galaxies in the DAS Main galaxy sample. We excluded from our sample those duplicate entries that have spectra of lower accuracy. We count as duplicate entries those galaxies, which have the projected separation less than $5 \mathrm{kpc}$ (see Paper II for more details). If both duplicates had a high redshift 
E. Tago et al.: SDSS DR7 volume-limited groups

Table 1. The SDSS DR7 Main DAS (March 2009) sample used and the FoF parameters for the flux- and volume-limited group catalogues.

\begin{tabular}{|c|c|c|c|c|c|c|c|c|c|}
\hline Sample & $M_{\text {lim }}$ & & & $N_{\text {gal }}$ & $N_{\text {groups }}$ & $N_{\text {single }}$ & $\begin{array}{c}L L_{r 0} \\
\mathrm{~km}^{-1} \mathrm{~s}^{-1}\end{array}$ & $\stackrel{L L_{p 0}}{h^{-1} \mathrm{Mnc}}$ & $\%$ in groups \\
\hline 1 & 2 & 3 & 4 & 5 & 6 & 7 & 8 & 9 & 10 \\
\hline $\begin{array}{l}\text { Flux-limited groups } \\
\text { DR7 Main } \\
\text { Volume-limited groups }\end{array}$ & 17.77 & 0.009 & .2 & 583362 & 78800 & 314524 & $250-579$ & $0.25-0.58$ & 46.1 \\
\hline DR7-18 & -18.0 & 0.02595 & 0.04590 & 40665 & 5463 & 24248 & 250.0 & 0.2500 & 40.4 \\
\hline DR7-19 & -19.0 & 0.02595 & 0.07141 & 94912 & 12590 & 58733 & 306.7 & 0.3067 & 38.1 \\
\hline DR7-20 & -20.0 & 0.02595 & 0.11012 & 148195 & 18973 & 96842 & 409.4 & 0.4094 & 34.7 \\
\hline DR7-21 & -21.0 & 0.02595 & 0.16801 & 103978 & 9139 & 82854 & 543.8 & 0.5438 & 20.3 \\
\hline
\end{tabular}

Notes. Columns: 1 - subsample name; 2 - galaxy magnitude limit in $r$-band (apparent for flux-limited groups, absolute for volume-limited groups); 3 - lower redshift limit; 4 - higher redshift limit; 5 - total number of galaxies $N_{\text {gal }}$ in samples; 6 - number of groups in samples $(n \geq 2) ; 7$ - number of single galaxies; $8-$ FoF linking length in the radial velocity, for $z=0 ; 9-$ FoF linking length in the projected distance in the sky, for $z=0$; 10 - fraction of galaxies in groups $(n \geq 2)$.

confidence parameter $\left(z_{\text {conf }}>0.99\right)$, we excluded the fainter duplicate. In total, we excluded 11490 duplicate galaxies from our sample.

The total number of galaxies was thus reduced to 583362 . The data on the final sample are presented in Table 1. The redshifts were corrected for the motion relative to the CMB. For linear dimensions we used the co-moving distances (see, e.g. Martínez \& Saar 2002), computed with the standard cosmological parameters: the Hubble parameter $H_{0}=100 h \mathrm{~km} \mathrm{~s}^{-1} \mathrm{Mpc}^{-1}$, the matter density $\Omega_{\mathrm{m}}=0.27$, and the dark energy density $\Omega_{\Lambda}=0.73$.

\section{Friends-of-friends algorithm}

Turner \& Gott (1976) were the first to use cluster analysis to search for groups of galaxies. This method was named the "friends-of-friends" (FoF) algorithm by Press \& Davis (1982), and promoted by Zeldovich et al. (1982) and by Huchra \& Geller (1982). In the FoF method, galaxies can be linked into systems with a certain linking length (or neighbourhood radius). Experience of numerous studies shows that the choice of the FoF parameters depends on the goals of the study. For example, while Weinmann et al. (2006) searched for more compact groups in the SDSS DR2 sample than the groups in this paper, applying strict criteria in the FoF method, Berlind et al. (2006) applied the FoF method to volume-limited samples of the SDSS with the goal to measure the group multiplicity function and to constrain dark matter halos. This reduced the incompleteness of the sample, but it also led to a lower number of groups. In this paper our goal is to obtain the SDSS DR7 groups for further determination of the luminosity density field and for studying the properties of the galaxy network. Hence, our goal is to find as many groups as possible to trace the supercluster-void network: within lowdensity regions, within filaments, within high-density peaks in the superclusters (Einasto et al. 2003b,c, 2007a,b).

Einasto et al. (1984) and Berlind et al. (2006) have concluded that it is not easy to find a suitable linking length even for a volume-limited sample of galaxies. The virialisation condition, a certain density contrast level, FoF methods, or other criteria do not work universally well for all density ranges of the galaxy distribution, due to the variable mean density of galaxies in different regions of space. Additional difficulties arise for flux-limited samples of galaxies where the linking length depends also on the distance from the observer. Eke et al. (2004) have given a summary of various methods to find clusters in galaxy samples.

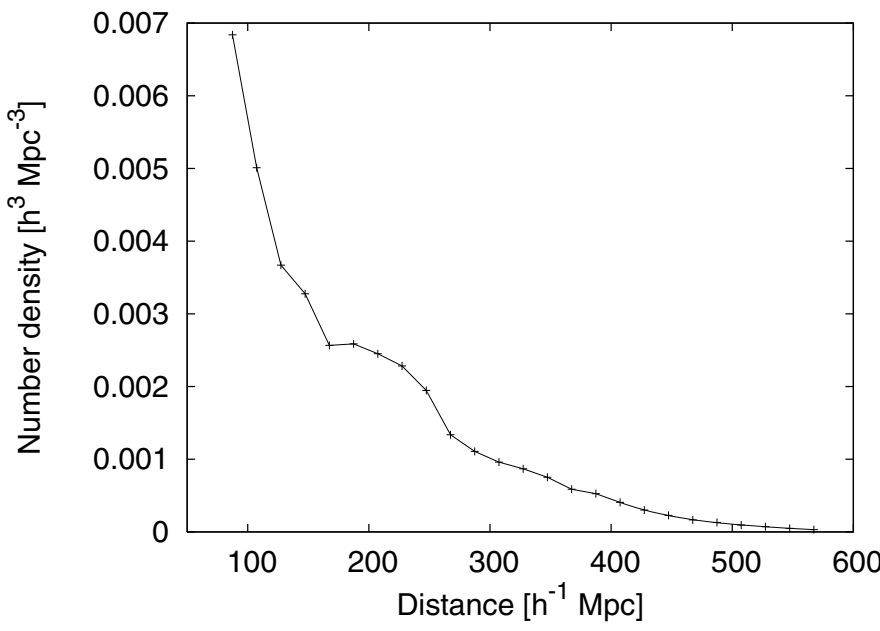

Fig. 2. The number density of groups in the SDSS DR7 flux-limited sample as a function of distance from the observer.

As shown in Papers I and II, it is actually the density of galaxies in groups, not in the general field, that determines the choice of group selection parameters. There exists a close correlation between luminosities of galaxies in groups and their positions within groups: bright galaxies are concentrated close to the centre, and less bright companions lie in the outskirts (for an early analysis of this luminosity-density correlation see Einasto et al. 1974, for a recent discussion see Paper I). In Papers I and II we found that while constructing group catalogues, a slightly increasing linking length with distance has to be used.

\section{Selection effects}

The main selection effects in group catalogues are caused by a fixed interval of apparent magnitudes in galaxy surveys.

The main consequence of this selection effect is the inhomogeneous spatial distribution of groups: the volume density of groups decreases with increasing distance. We show this in Fig. 2, where we plot the mean number density of groups as a function of distance. Note that the local maximum of the number density of groups at a distance of $\approx 220 h^{-1} \mathrm{Mpc}$ is due to a very rich supercluster SCL126 (Einasto et al. 1997), also called the Sloan Great Wall. 


\section{Scaling of the linking length}

Sizes of groups depend directly on the choice of the linking length $L L$, or more generally on its scaling law. Strong selection effects can be observed here.

In the majority of recent papers dedicated to group searches, the group finders are tuned with mock $N$-body catalogues (Eke et al. 2004; Yang et al. 2005). The mock group catalogues are homogeneous and all parameters of the mock groups can be easily found and applied to searches for real groups. Still, mock groups are only an approximation of the real groups, using model galaxies in dark matter halos. As we have noted, it is difficult to properly model the luminosity-density correlation found in real groups.

At large distances from the observer, only the brightest cluster members are visible, and these brightest members form compact cores of clusters, with sizes much less than the true size of the clusters.

For this reason we have proposed to use observed groups to calibrate the scaling of group properties with distance. This procedure is described in detail in Paper I. We here present the method briefly and give the results for the SDSS DR7 groups.

We created test group catalogues from the SDSS DR7, selecting in the nearby volume $d<200 h^{-1}$ Mpc all rich groups (with multiplicity $n \geq 20$ ). We included both constant and variable linking lengths to create the test groups; the final results did not depend on this choice. The values of the FoF parameters are given in Table 1 and Sect. 8.1. Assuming that the group members are all at the mean distance of the group, we determined their absolute magnitudes and peculiar radial velocities. Then we shifted these nearby groups, calculating the parameters of the groups (new $k$-corrections and apparent magnitudes for the group members), as if the groups were located at larger distances. We increased the distances step by step (using a $\Delta z=0.001$ step in redshift). As with the increasing distance more and more fainter members of groups fall outside the observational window of apparent magnitudes, the group membership changes. We then determined new properties of the groups - their multiplicities, characteristic sizes, rms velocities, and number densities. We also calculated the minimum FoF linking length necessary to keep the group together at this distance. To determine that, we built the minimal spanning tree (MST) for the group (see, e.g. Martínez \& Saar 2002) and found the maximum length of the MST links.

As the original groups had different sizes and initial redshifts, we found the relative changes of their properties with respect to the redshift change. The individual linking lengths have a substantial scatter. In Fig. 3 we show the variation of the mean linking length and its rms error with the redshift change.

We determined the mean values of the group linking lengths in $\Delta z=0.001$ redshift bins (the step we used for shifting the groups). We found that the scaling is not sensitive to the richness of the test group; in order to extend the scaling to larger redshifts we included nearby groups with a richness $n \geq 20$ as test groups. The scaling law for the SDSS is moderately different from the scaling law found for the 2dFGRS groups in Paper I, but it can still be approximated by a slowly increasing arctan function. Due to the narrow magnitude window of the SDSS, at higher values of $z$ only compact cores of groups (or binary galaxies) are found. This causes deviation from the scaling law, starting at the redshifts, above which most groups retain only their compact cores, where the brightest group galaxies reside. Therefore, the behaviour of the scaling law is also a test for the redshift of the homogeneity limit of the group catalogue (in our

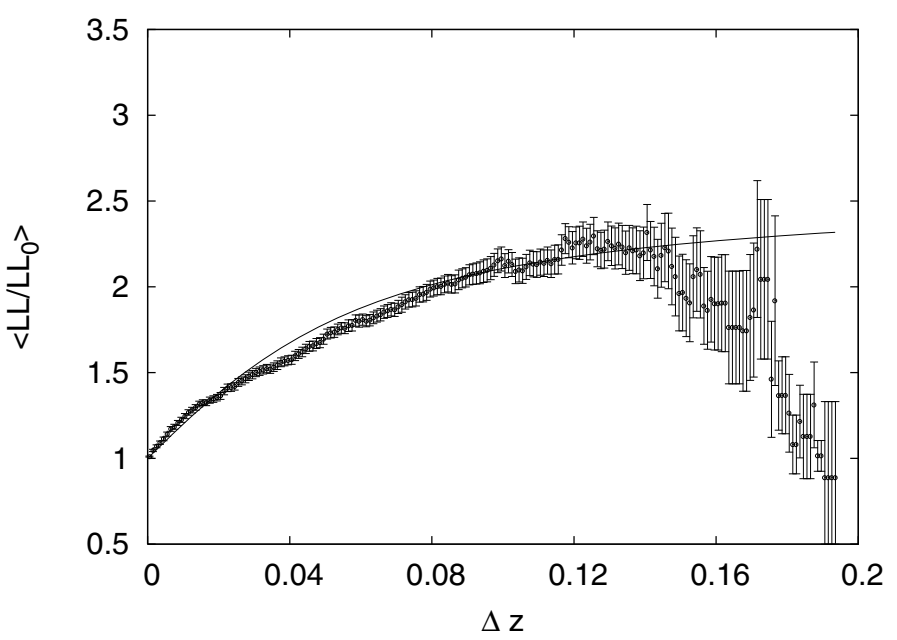

Fig. 3. Scaling of the group FoF linking length with redshift for the flux-limited test sample. The ordinate is the mean ratio of the minimal linking length $L L$ at a redshift $z$ necessary to keep the group together to the original linking length $L L_{0}$ that defined the group at its initial redshift $z_{0}$; error bars are the rms errors obtained from the $L L$ of individual groups; the abscissa is the redshift difference $\Delta z=z-z_{0}$. Solid line represents the scaling law given by Eq. (1).

case $z \approx 0.14$ or the co-moving distance $\sim 400 h^{-1} \mathrm{Mpc}$ ). A good parametrisation of the scaling law for the linking length is

$L L / L L_{0}=1+a \arctan \left(z / z_{\star}\right)$,

where $a=1.00, z_{\star}=0.050$ and $L L_{0}$ is the value of $L L$ at initial redshift. As the test groups all lie at small redshifts, we can replace the redshift change $\Delta z$ by the redshift itself.

The main difference between the scaling laws of the DR7 and $2 \mathrm{dFGRS}$ groups lies in the validity range. This is due to different magnitude limits in these flux-limited samples. The selection of initial groups does not influence much the scaling of their properties with distance. We tested this using three different initial scaling laws to select the test groups: two with a constant $L L$ and one with a variable $L L$. The final scaling relation practically did not depend on the initial group selection (i.e. on the initial scaling law).

\section{Volume-limited samples of groups of galaxies in the SDSS DR7}

A flux-limited sample suffers by its nature for selection effects, although attempts have been made to minimise them. An advantage of such a sample is the maximum use of observational data. However, there are studies, which need to create volumelimited samples, involving rejection of a large amount of data. One of these cases is the comparison of an observed sample with $\mathrm{N}$-body simulations, in particular with dark matter halo models with sub-halos representing galaxies. To create a volume-limited sample we have to set an absolute magnitude (luminosity) limit for a galaxy sample and have to reject less luminous galaxies.

In the case of groups of galaxies an additional problem arises. Inclusion of galaxies in volume-limited samples depends on their absolute magnitude. The latter depends on the distance of a galaxy. At first, when we have not yet built groups, we have to determine the absolute magnitude of a galaxy on the basis of its redshift. When we have built groups, the distance of a group member galaxy is determined by the group mean radial velocity (redshift). This demands the recalculation of the absolute magnitudes of the member galaxies, and the new magnitudes may 


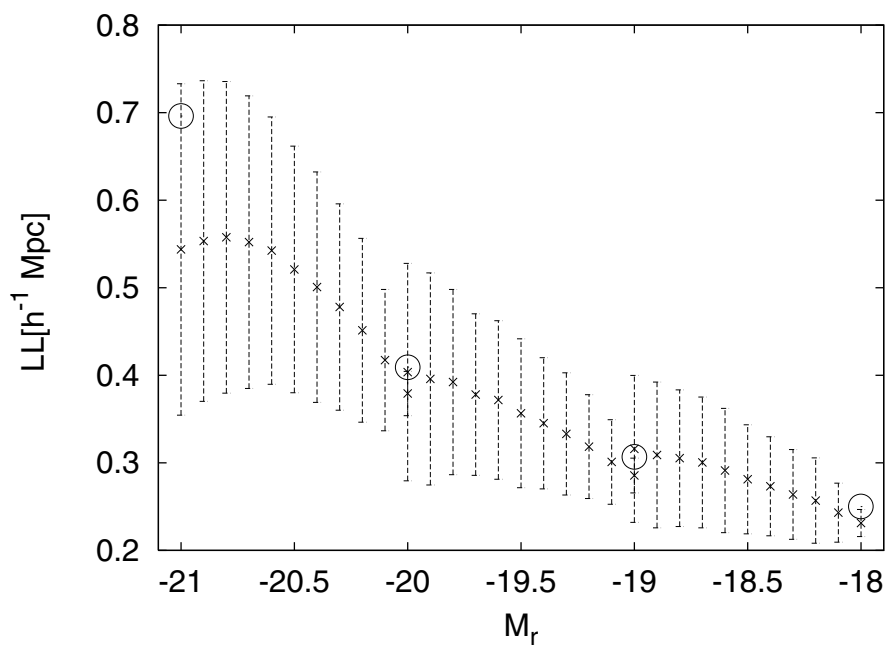

Fig. 4. Scaling of the linking length $L L$ for four volume-limited subsamples, corresponding to the magnitude limits from $M_{r}<-21$ to $M_{r}<-18$. Lines with error-bars show the distribution of the minimum linking lengths needed to keep the sub-sample groups together when the sample faint magnitude limit was changed. Crosses show the mean $L L$, error bars - the rms deviations. The linking lengths used are those given by the crosses for the four faint sub-sample limits $\left(M_{r}=-18,-19,-20,-21\right)$. For comparison, we show the $L L$ for every sub-sample, determined by the number density scaling (open rings).

happen to be outside the sample magnitude limits. These galaxies should be deleted from the sample, and groups should be built again. After that, we have to recalculate the absolute magnitudes, based on the new group data. Nothing guarantees that this iteration will ever stop.

We applied the following procedure to overcome the problem. At first we determined groups of galaxies in the full fluxlimited sample, using FoF together with a scaled $L L$ (by the arctan law as found in Sect. 5). With these groups, we calculated the distances and absolute magnitudes of their member galaxies. Then we exclude from the groups the galaxies, which do not satisfy the criteria of a given volume-limited sample. Next, we apply the FoF procedure with a constant $L L$ to the sub-sample of galaxies we found. This step completes the group searching procedure.

We have applied this procedure for four subsamples with various absolute magnitude limits in the $r$-band $\left(M_{r}=\right.$ $-18,-19,-20,-21)$. A constant $L L$ (not depending on distance) was used for each volume-limited sub-sample.

As different volume-limited subsamples have different densities, the linking length $L L$ used to build groups has to be different. As the usual number-density scaling $\left(L L \sim n^{-1 / 3}\right)$ does not account for the luminosity-density relation, we found the $L L$ for the volume-limited subsamples as we did for the flux-limited samples, using the observed groups. This time we did not shift the groups in distance, but we applied different absolute magnitude limits to the group galaxies and found the minimum $L L$ that would keep the groups together. Figure 4 presents the scaling of $L L$ with the bright absolute magnitude limit in the four subsamples (the mean and one-sigma range). The circles show the $L L$ found for the number-density scaling. The proper linking lengths are those for the faint sample limit; the intermediate magnitude points illustrate the scaling of $L L$ with magnitude.

We see that in two fainter subsamples the $L L$ scales as predicted by the number-density scaling, i.e. the luminosity-density relation is not important here. But the linking length for the

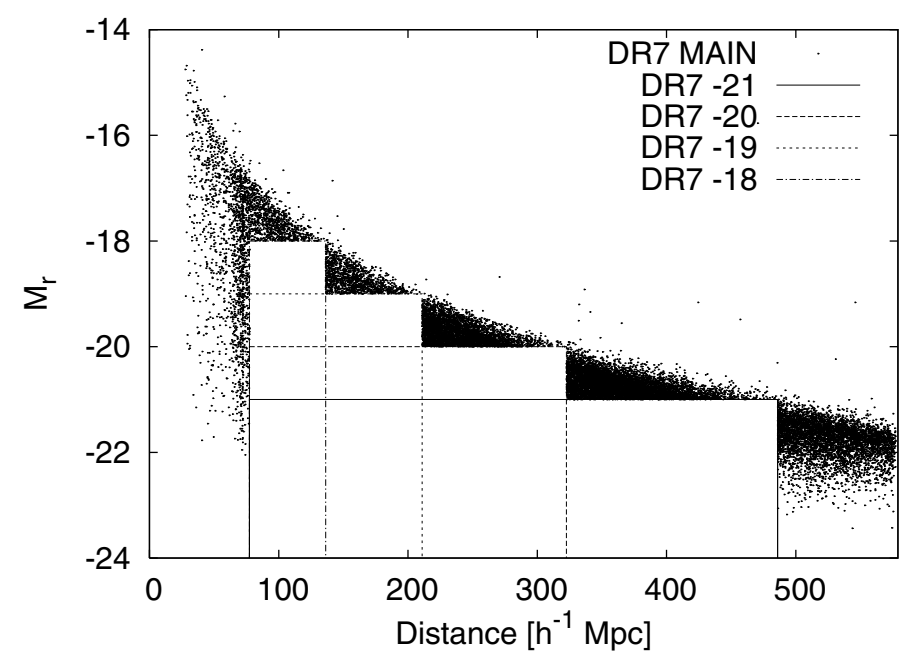

Fig. 5. Four volume-limited subsamples in the distance-absolute magnitude plot corresponding to the magnitude limits $M_{r}=-18,-19,-20$, and -21 . For clarity, only limits of subsamples and the excluded main sample galaxies are plotted.

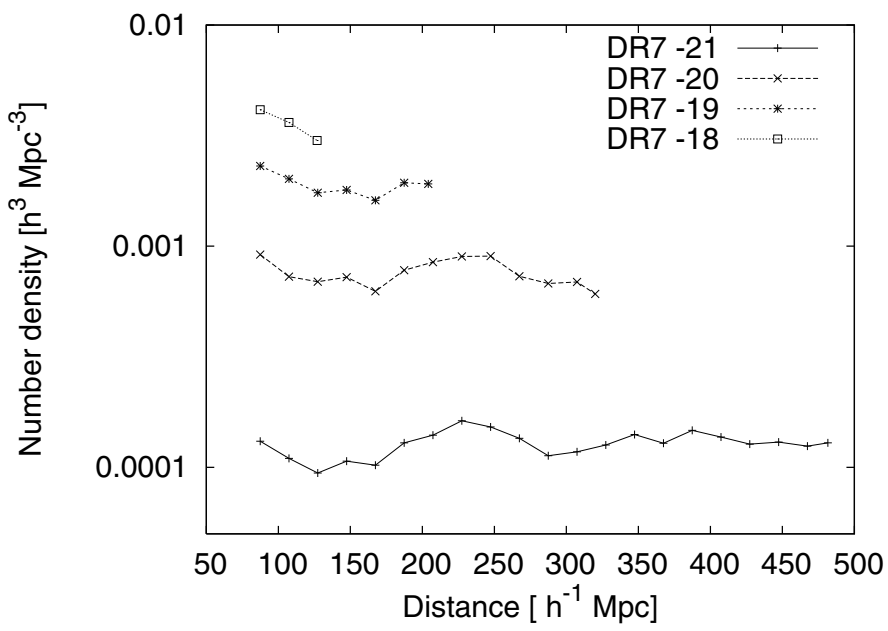

Fig. 6. Spatial number density of groups in four volume-limited subsamples corresponding to the absolute magnitude limits $M_{r}=$ $-18,-19,-20,-21$ as a function of distance.

brightest sub-sample $\left(M_{r}<-21.0\right)$, found from the second brightest sample $\left(M_{r}<-20.0\right)$, is considerably smaller than the $L L$ found by the number density scaling. This confirms that the luminosity-density relation is most prominent among the bright galaxies in group centres. We used the mean linking lengths given by the crosses for the faint sub-sample limits $\left(M_{r}=-18,-19,-20,-21\right)$.

The FoF parameters and results for each sub-sample are presented in Table 1. The calculation of absolute magnitudes is presented in Sect. 9.2.

Figure 5 shows the volume-limited samples in a distanceabsolute magnitude plot. Closer than $z=0.026$ the bright galaxy content is incomplete. The fraction of galaxies in all four volume-limited samples is $66.7 \%$ (from the full magnitudelimited sample) and the fraction of common galaxies (the fraction of galaxies included at least in two subsamples of four) is $34.3 \%$. Table 1 describes the samples.

To check how well we have built the volume-limited subsamples, we show in Fig. 6 the dependence of the spatial number density of groups on the distance for all four subsamples. There 
Table 2. The first rows of the SDSS DR7 flux-limited group catalogue described in this paper.

\begin{tabular}{|c|c|c|c|c|c|c|c|c|c|c|}
\hline $\mathrm{ID}_{\mathrm{gr}}$ & $n$ & $\begin{array}{c}\mathrm{RA} \\
{[\mathrm{deg}]}\end{array}$ & $\begin{array}{c}\text { Dec } \\
{[\mathrm{deg}]}\end{array}$ & $\begin{array}{c}\text { Dist } \\
{\left[h^{-1} \mathrm{Mpc}\right]}\end{array}$ & $\begin{array}{c}\text { Size }_{\text {sky }} \\
{\left[h^{-1} \mathrm{Mpc}\right]}\end{array}$ & $\begin{array}{c}\sigma_{R} \\
{\left[h^{-1} \mathrm{Mpc}\right]}\end{array}$ & $\begin{array}{c}\sigma_{V} \\
{\left[\mathrm{~km} \mathrm{~s}^{-1}\right]}\end{array}$ & $\begin{array}{c}R_{\mathrm{vir}} \\
{\left[h^{-1} \mathrm{Mpc}\right]}\end{array}$ & Weight & $\begin{array}{c}L_{\text {obs }} \\
{\left[10^{10} h^{-2} L_{\odot}\right]}\end{array}$ \\
\hline 1 & 2 & 3 & 4 & 5 & 6 & 7 & 8 & 9 & 10 & 11 \\
\hline 1 & 3 & 109.822853 & 41.162451 & 83.7400 & 0.0634 & 0.037494 & 131.052963 & 0.050429 & 1.056 & 0.2402961 \\
\hline 2 & 2 & 109.960762 & 41.369118 & 263.6165 & 0.3486 & 0.226603 & 15.567180 & 0.320465 & 1.534 & 1.375062 \\
\hline 3 & 4 & 110.053011 & 41.275907 & 195.5326 & 0.5412 & 0.227071 & 194.728271 & 0.279245 & 1.263 & 1.713846 \\
\hline 4 & 4 & 110.290878 & 41.030225 & 71.1812 & 0.5744 & 0.230941 & 126.573807 & 0.229455 & 1.072 & 0.1092000 \\
\hline 5 & 2 & 110.125167 & 41.206594 & 132.8659 & 0.0448 & 0.030350 & 19.526409 & 0.042921 & 1.115 & 0.3151180 \\
\hline
\end{tabular}

are no global trends in the spatial densities within the subsamples that confirm their homogeneity. An exception is the $M_{r}=-18$ sub-sample; this nearby and narrow sub-sample is most influenced by incompleteness in bright galaxies.

\section{From the initial catalogue to volume-limited samples}

In this section we describe in detail the steps necessary to get from the initial catalogues obtained from the SDSS DR7 web site to the volume-limited samples of groups of galaxies.

Input: SDSS incremental releases from DR1 to DR7 of the main galaxy DAS samples in FITS format, compressed into files maingals-DR123a.fit.gz (and DR123b, DR4, DR5, DR6, DR7, respectively).

1. Extraction of the galaxy sample:

1.1 extraction of the necessary columns from the incremental catalogues, and concatenation of them into a full DR7 catalogue;

1.2 rejection of duplicate galaxies;

1.3 rejection of a few galaxies with undetermined magnitudes and/or magnitude errors;

1.4 selection of galaxies from the main footprint in the $\lambda$ and $\eta$ coordinates, redshift, and $r$-band magnitude ranges (see in Table 1 and Sect. 2).

2. Creation of the flux-limited group sample:

2.1 performing the initial FOF procedure, using a constant $L L$;

2.2 determination of the $L L$ scaling law, using sample shifting and the minimal spanning tree method that takes into account the luminosity-density relation in groups;

2.3 searching for the final flux-limited sample of groups, using a modified FoF with a changing $L L$, according to the scaling law;

2.4 application of the $k$ - and $e$-corrections to find the absolute magnitudes and luminosities for the flux-limited sample of galaxies and groups.

3. Creation of volume-limited subsamples of groups:

3.1 determination of the limits of the volume-limited subsamples in redshift, absolute and apparent magnitudes of galaxies;

3.2 search of volume-limited subsamples of groups of galaxies applying FoF with a constant $L L$ within a subsample, while scaling $L L$ between subsamples, using the luminosity-density scaling (see Sect. 6).

Output: catalogues of galaxies and of groups for the fluxlimited sample and for each volume-limited sub-sample.

\section{Group catalogue}

\subsection{The group finder}

We adopt the scaling of the linking length found above, but we have to select the initial values for the linking length.

In order to find the best initial linking lengths, we tried a number of different parameter values. Referring to the arguments presented in Papers I and II we accepted the size ratio of the radial to the transversal linking lengths to be 10 (if the radial linking length in $\mathrm{km} \mathrm{s}^{-1}$ is transformed into a formal "distance" in $h^{-1} \mathrm{Mpc}$ ). We chose the initial values $L L_{r 0}=250 \mathrm{~km} \mathrm{~s}^{-1}$ and $L L_{p 0}=0.25 h^{-1} \mathrm{Mpc}$. Higher values for $L L_{p}$ lead to inclusion of galaxies from neighbouring groups and filaments. Lower values for $L L_{r}$ exclude the fastest members in intermediate richness groups.

The selected parameters lead to reasonable group properties. However, a closer inspection shows that four of our rich groups have a richness much larger $(n>300)$ than the rest (group ID: $73044,72185,74853$, and 31802 ). These are the well-known nearby rich Abell clusters: A1656 (Coma), A2151/2152(Hercules), A2197/2199, and A1367, respectively. We consider these clusters as exceptions.

\subsection{The final flux-limited and volume-limited catalogues}

Our final flux- and volume-limited catalogues (Table 2) include groups with richness $\geq 2$. As an example we present here the first lines of our group table that includes the following columns for each group:

1) group identification number;

2) group richness (the number of member galaxies);

3) RA (J2000.0) in degrees (the mean of member galaxies);

4) Dec (J2000.0) in degrees (the mean of member galaxies);

5) group distance in $h^{-1} \mathrm{Mpc}$ (the mean co-moving distance for member galaxies, corrected for the movement with respect to the CMB);

6) maximum projected size (in $h^{-1} \mathrm{Mpc}$ );

7) rms deviation of projected distance in the sky from the group centre (in $h^{-1} \mathrm{Mpc}$ );

8) rms radial velocity $\left(\sigma_{V}\right.$, in $\left.\mathrm{km} \mathrm{s}^{-1}\right)$;

9) virial radius in $h^{-1} \mathrm{Mpc}$ (the projected harmonic mean);

10) luminosity weight to correct for probable group member galaxies outside the survey visibility window;

11) observed total luminosity of the group $\left(10^{10} h^{-2} L_{\odot}\right)$.

An identification number is attached to the groups in the ascending order of the RA coordinate. The calculation of luminosities is described in Sect. 9.2.

We also give (in electronic form) a catalogue of all individual galaxies along with their group identification number and the group richness, ordered by the group identification number, 


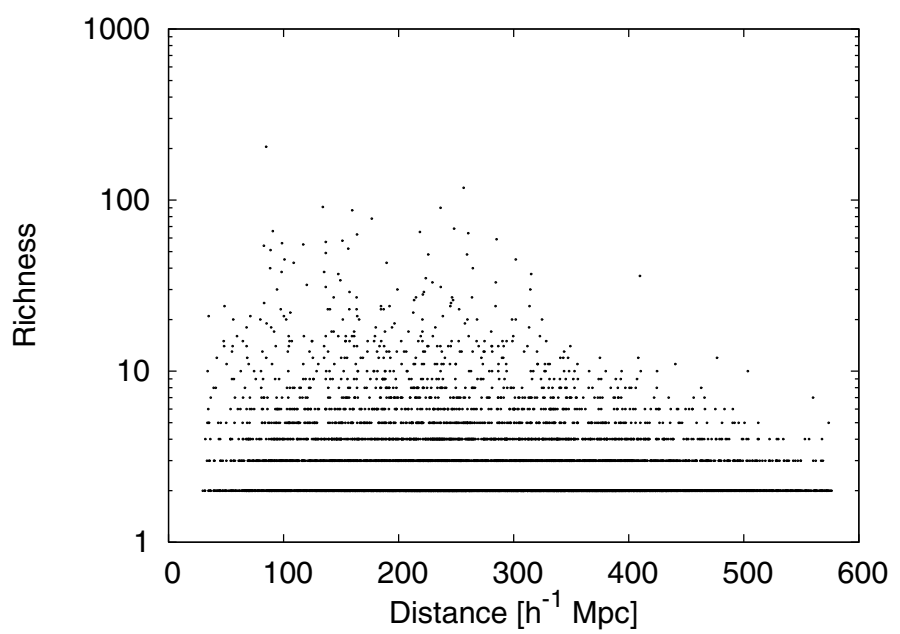

Fig. 7. Richness of groups in the flux-limited sample as a function of distance.

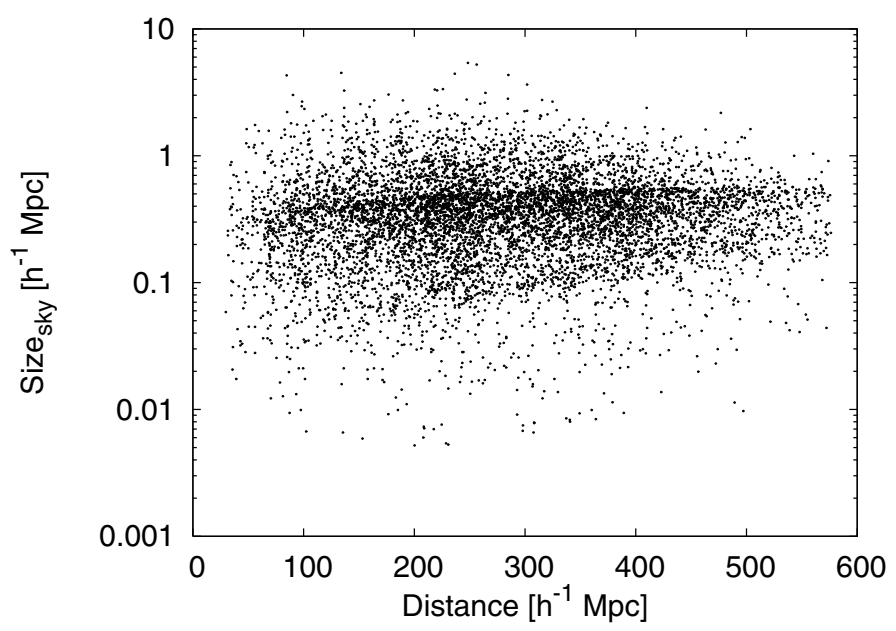

Fig. 8. Maximum size of groups in the sky projection in the flux-limited sample as a function of distance.

to facilitate search. The tables of galaxies end with a list of the most isolated galaxies (small groups with only one bright galaxy within the observational window of magnitudes); their group identification number is 0 and group richness is 1 .

\section{Properties of flux-limited groups}

\subsection{Multiplicities, sizes and rms velocities of groups}

Here we present in short the main properties of our flux-limited groups. In Fig. 7 we show the multiplicity of groups (the number of member galaxies) as a function of distance from the observer for the SDSS DR7. Due to the use of an apparent magnitude limited sample the richness (multiplicity) of groups depends on the distance.

We see that rich groups with at least 30 member galaxies are seen only up to a distance of about $300 \mathrm{~h}^{-1} \mathrm{Mpc}$, thereafter the mean multiplicity decreases considerably with distance. This selection effect must be accounted for in the multiplicity analysis.

We define the size of a group as the largest projected distance between galaxies within the group. In Fig. 8 we see that the sizes of the largest groups slightly increase with distance up to $d=250 h^{-1} \mathrm{Mpc}$, and thereafter slowly decrease. This decrease is expected since in more distant groups only bright

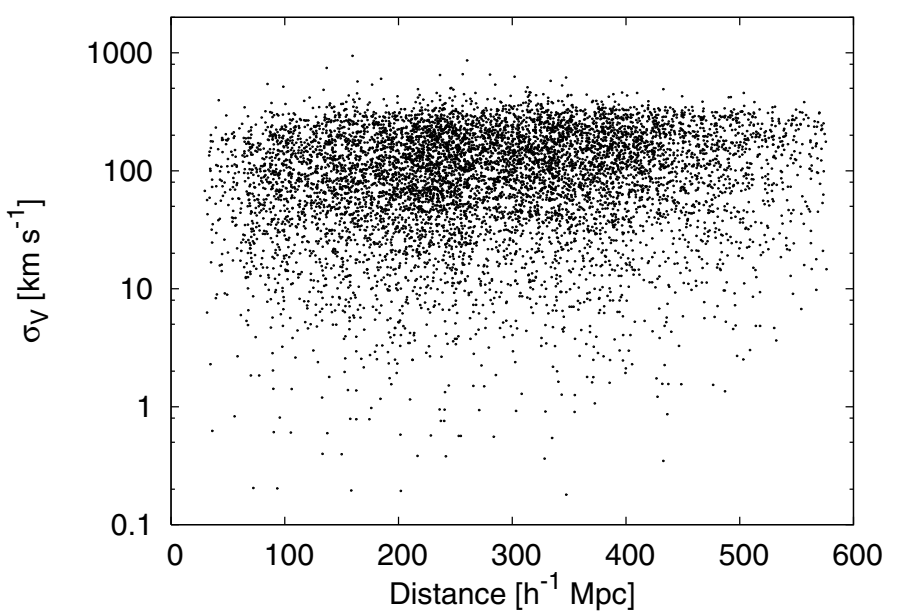

Fig. 9. Rms velocities of galaxies in groups in the flux-limited sample as a function of distance.

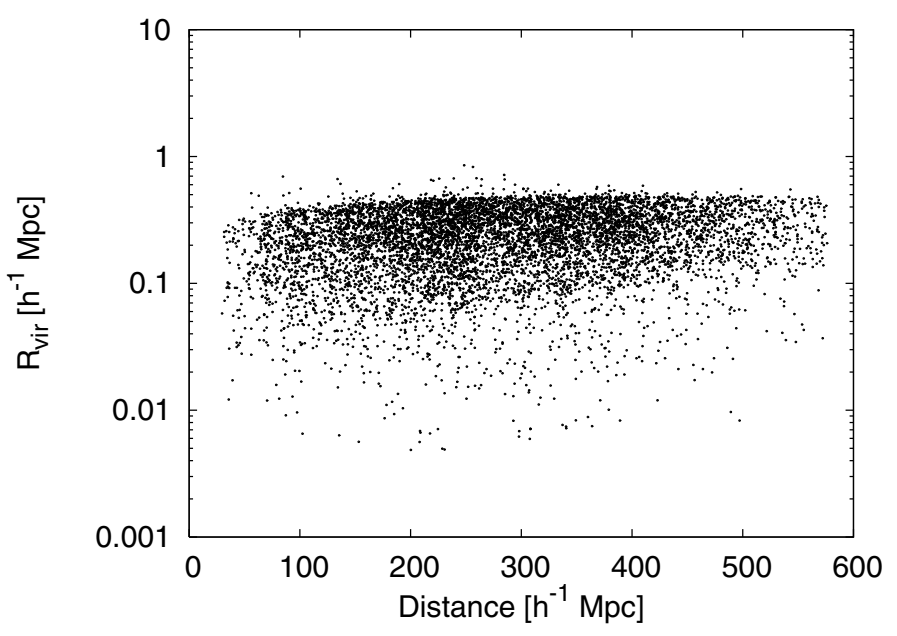

Fig. 10. Virial radius of groups in the flux-limited sample as a function of distance.

galaxies are seen, and they form the compact cores of groups. This is partly due to the absence of nearby bright galaxies in the SDSS sample, and partly because the under-dense region behind the Local Supercluster is populated by less rich, smaller groups (see Einasto et al. 2003b, about environmental enhancement of groups). Relatively sharp edges in Figs. 8-10 are caused by groups of low richness (doubles and triples).

Groups of low multiplicity, in particular double and triple galaxies, probably are not all genuine groups of galaxies. For example, the estimated velocity dispersions of these systems are severely biased. A large fraction of these groups may have fainter galaxies with luminosities outside of the visibility window. They may be cores of distant richer groups. If these groups were at smaller distances, their fainter galaxies could have been observed.

The velocity dispersions $\sigma_{V}^{2}$ for our groups were calculated with the standard formula

$\sigma_{V}^{2}=\frac{1}{\left(1+z_{\mathrm{m}}\right)^{2}(n-1)} \sum_{i=1}^{n}\left(V_{\mathrm{i}}-V_{\text {mean }}\right)^{2}$

where $V_{\text {mean }}$ and $z_{\mathrm{m}}$ are the mean group velocity and redshift, respectively, $V_{i}$ is the velocity of an individual group member, and $n$ is the number of galaxies with observed velocities in a group. The need for the cosmological factor $1+z_{\mathrm{m}}$ is well 
explained by Harrison (1974). The rms velocities of galaxies in groups are plotted in Fig. 9.

Figure 10 shows the virial radii $R_{\text {vir }}$ of the flux-limited groups, calculated by the formula

$$
\frac{1}{R_{\mathrm{vir}}}=\frac{2}{\left(1+z_{\mathrm{m}}\right) n(n-1)} \sum_{i \neq j}^{n} \frac{1}{R_{i j}},
$$

where $R_{i j}$ is the projected distance between galaxies in pairs in a group.

As the properties of groups are independent of distance these figures show that the selection effects were properly taken into account when generating our flux-limited group catalogue.

\subsection{Luminosities of groups}

The limiting apparent magnitudes of the complete sample of the SDSS catalogue in $r$-band are 12.5 and 17.77. The faint limit actually fluctuates slightly from field to field, but in the present context we shall ignore that. We regard every galaxy as a visible member of a group or cluster within the visible range of absolute magnitudes, $M_{1}$ and $M_{2}$, corresponding to the observational window of apparent magnitudes at the distance of the galaxy. To calculate the total luminosities of groups we have to take into account possible members outside the visibility window. This estimated total luminosity of groups is calculated as follows:

$L_{\mathrm{tot}}=W_{L} \sum_{i=1}^{n} L_{\mathrm{obs}, i}$,

where $L_{\mathrm{obs}, i}=L_{\odot} 10^{0.4\left(M_{\odot}-M\right)}$ is the luminosity of a galaxy of an absolute magnitude $M$ and $M_{\odot}$ is the absolute magnitude of the Sun, and

$W_{L}=\frac{\int_{0}^{\infty} L \phi(L) \mathrm{d} L}{\int_{L_{1}}^{L_{2}} L \phi(L) \mathrm{d} L}$

is the luminosity weight (the ratio of the expected total luminosity to the expected luminosity in the visibility window). The sum in (4) includes all group members, and $n$ is the group richness. In the last equation $L_{1,2}=L_{\odot} 10^{0.4\left(M_{\odot}-M_{1,2}\right)}$ are the luminosity limits of the observational window, corresponding to the absolute magnitude limits of the window $M_{1,2}$ :

$M_{1,2}=m_{1,2}-25-5 \log _{10}\left(d_{L}\right)-K_{d_{L}}$.

Here $d_{L}=d(1+z)$ is the luminosity distance, $d$ is the co-moving distance in units of $h^{-1} \mathrm{Mpc}$, and $z$ is the observed redshift; the luminosity limits of the observational window are $m_{1}=17.77$ and $m_{2}=12.5$; the term $K_{d_{L}}$ is the average $k+e$-correction for the given luminosity distance $d_{L}$.

In the calculation of weights (5) we assumed that galaxy luminosities are distributed according to a double power-law function that was introduced in the past, e.g. Christensen (1975), Kiang (1976), Abell (1977), Mottmann \& Abell (1977):

$\phi(L) \mathrm{d} L \propto\left(L / L^{*}\right)^{\alpha}\left(1+\left(L / L^{*}\right)^{\gamma}\right)^{(\delta-\alpha) / \gamma} \mathrm{d}\left(L / L^{*}\right)$,

where $\alpha, \gamma, \delta$, and $L^{*}$ are parameters. We used the double powerlaw rather than the Schechter function, as it gives a better fit for the observed galaxy luminosity function, especially at its highluminosity end (Tempel et al. 2009). We used the double powerlaw function with the parameters $\alpha=-1.06, \gamma=1.058, \delta=$ $-17.74, L^{*}=13.80$. We included all the SDSS main galaxies

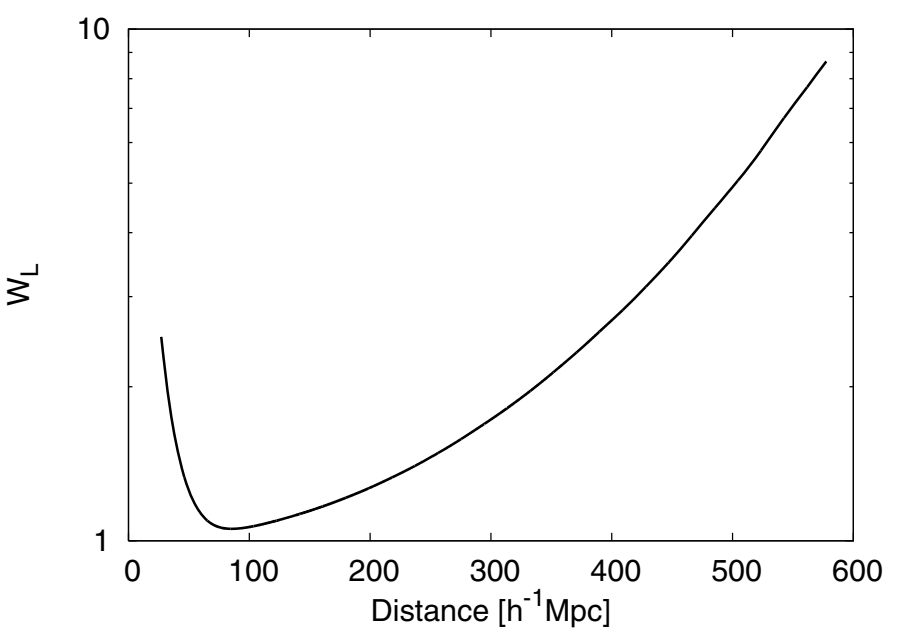

Fig. 11. Weights used to correct for probable group members outside the observational luminosity window.

(galaxies in groups and the most isolated galaxies) to find the luminosity function. A more detailed explanation of the double power-law function and how we derived the parameters is given in our paper on the $2 \mathrm{dFGRS}$ luminosity function (Tempel et al. 2009).

The $k$-correction for the SDSS galaxies was calculated with the KCORRECT algorithm (Blanton et al. 2003a; Blanton \& Roweis 2007). We also accepted $M_{\odot}=4.53$ (in the $r$ photometric system). The evolution correction $e$ was calibrated according to Blanton et al. (2003b).

We give for each group the total observed luminosity and the luminosity weight.

The luminosity weights for the groups of the SDSS DR7 are plotted as a function of the distance from the observer in Fig. 11. We see that the mean weight is slightly higher than unity in a distance interval $\approx 70-100 \mathrm{~h}^{-1} \mathrm{Mpc}$, and increases both toward smaller and larger distances. The increase at small distances is due to missing very bright member galaxies of groups, which lie outside the observational window, and at large distances the increase is caused by the absence of faint galaxies. The weights grow fast for nearby groups and for groups farther away than about $400 h^{-1} \mathrm{Mpc}$. At these distances the correction factors start to dominate and the luminosities of the groups become uncertain.

\section{Discussion and conclusions}

Groups of galaxies are natural environments where galaxies form. Therefore, their properties are of high importance to understand the processes of galaxy formation. However, the search for groups of galaxies and evaluation of properties of obtained groups is yet surprisingly difficult. In this section we briefly discuss a few of the most general problems of our samples of groups of galaxies and note some possible caveats. A more detailed analysis of the properties of groups and comparison of various group catalogues will be carried out in separate papers.

\subsection{Problems of the sample creation of groups}

Our main goal is to determine the luminosity and density fields at large scales and to study large scale structure properties. Therefore, our attempts have been directed to obtain samples of groups of galaxies as extensive and homogeneous as possible. 


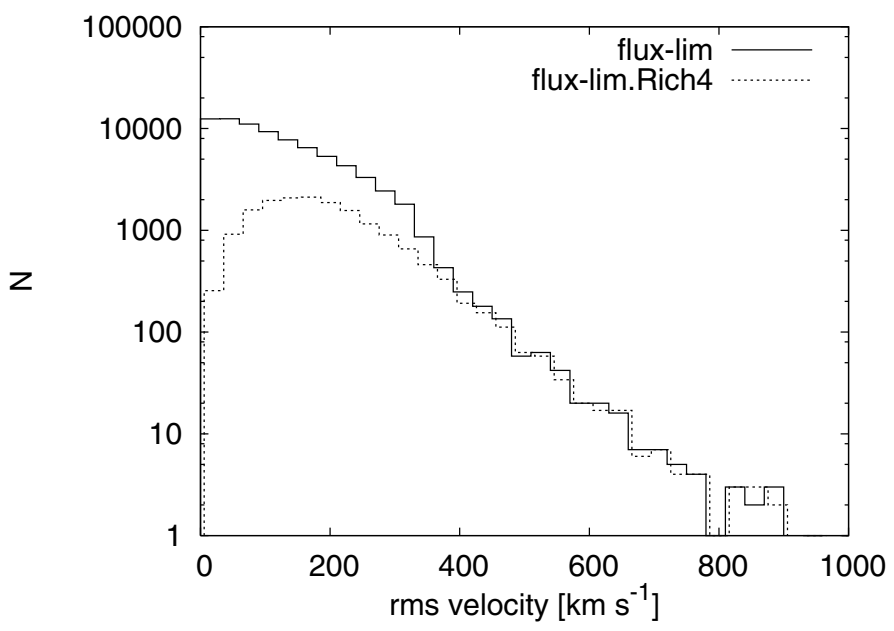

Fig. 12. Distribution of the rms velocities for the flux-limited sample: for all groups, and for the sample where low-richness groups $(n<4)$ are excluded.

This purpose can be achieved by taking the most extensive flux-limited sample of galaxies with measured redshits, in our case - the vast majority of the original catalogue SDSS DR7. The price of this choice are the selection effects in the fluxlimited sample of groups. We take selection effects into account by applying a refined scaling of the FoF group-finder search parameters. Using observed groups to find the $L L$ scaling law we take the properties of observed groups (the luminosity-density relation) into account. This yields one of the most homogeneous and extensive catalogues of groups.

The group search method described in this paper includes some problems that have to be noted.

First, the derived $L L$ law cannot be used for groups of all richness. As noted in Sect. 8, for very rich groups (i.e. rich Abell type clusters) the used $L L$ yields enormously large membership and size, as well as a multicomponent structure. Obviously, rich groups of galaxies have to be searched for with different search parameters or other methods (see, e.g. Aguerri et al. 2007; Popesso et al. 2007) than those employed for poor and medium rich groups.

Inhomogeneous samples of groups of galaxies could be created in another way. Consider the next case as an example of possible caveats in large samples of groups of galaxies. As we see in Figs. 6 and 9, in our samples the properties of groups almost do not depend on distance (redshift), which is a by-product of our $L L$ scaling procedure. Thus distance-dependent selection effects have been taken into account properly, if fine-tuning of the parameters for group search is done.

However, one has to select group samples for analysis carefully. As an example, we show in Fig. 12 the distribution of the rms velocities of groups from the flux-limited sample. This figure shows that if we exclude low-richness groups from the sample (for which the rms velocity is not reliable), then the rms velocity distribution is quite different from that of the whole sample, because there are many more small groups with small rms velocities than in the sample of groups with at least four member galaxies. Thus in studies of groups the proper choice of samples is important. To avoid contamination in their study Tovmassian \& Plionis (2009) selected a sample of groups in a narrow richness interval to study the dynamics of poor groups.

As an alternative way to obtain homogeneous samples of groups, we used volume-limited samples. The price of this selection is the exclusion of the majority of galaxies from the original

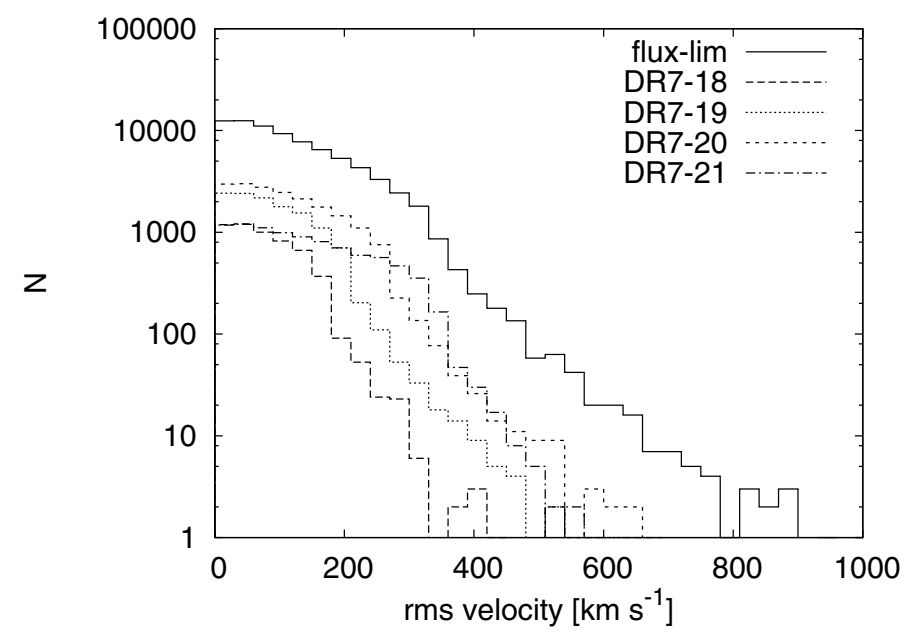

Fig. 13. Distribution of the rms velocities for the flux- and volumelimited samples.

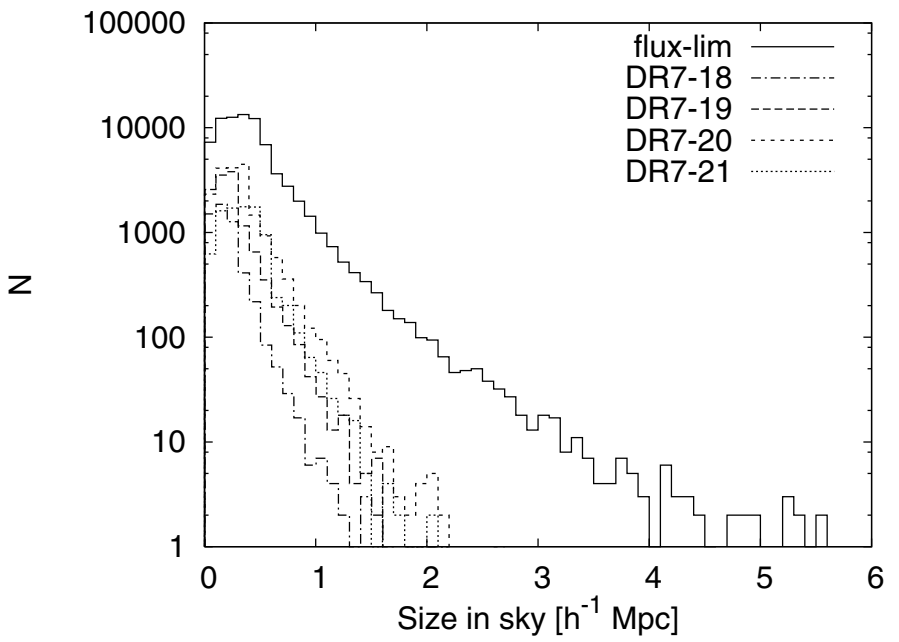

Fig. 14. Distribution of the projected sizes of groups in the sky for the flux- and volume-limited samples.

catalogue. However, in some cases the use of volume-limited sample is necessary (e.g., comparison of observed groups with numerical simulations). Scaling of the linking length for the volume-limited samples plays another role than for the fluxlimited ones: here scaling enables us to compare volume-limited subsamples of different absolute magnitude limits with each other. As noted in Sect. 6, strictly speaking, a clean volumelimited sample of groups cannot be created. We have overcome the problem as described in that section, but it remains unsolved in general.

In Figs. 13 and 14 we show the distribution of the rms velocities of groups and of projected sizes of groups from the flux- and four volume-limited samples of groups. These figures show that in volume-limited group samples the rms velocities of groups as well as their projected sizes have very simIlar distributions, and different only by sample sizes.

Besides the problems with the low richness groups there are problems with isolated galaxies, too. The criteria for the extraction of single galaxies depend on the goals of the authors and on the initial catalogues. The classical catalogue of isolated galaxies by Karachentseva (1973) was a well-defined catalogue a few decades ago. More recent studies by the AMIGA team (see, for example, Verley et al. 2007) have improved this catalogue. 
However, a reliable comparison of the isolated galaxies in our group catalogues with Karachentseva's catalogue is difficult, as they have different spatial volumes and because of the selection effects for bright SDSS galaxies.

We found the number of Karachentseva's isolated galaxies (CIG) which could belong to our groups or coincide with our most isolated galaxies. The number of the CIG galaxies in the common volume with our groups is about 409 (the remaining 642 CIG galaxies are outside this volume). 75 CIG galaxies can be identified with galaxies which belong to our groups. 154 CIG galaxies can be identified with our most isolated galaxies (or with groups consisting of one observed galaxy. About 180 CIG galaxies cannot be identified in our SDSS sample.

Studies of catalogues of fainter magnitude limits suggest that practically all galaxies belong to groups of galaxies, although in many cases the companion galaxies are faint and/or dwarf ones (e.g., as in the Local Group and in the M 81 group). In more distant galaxy samples we are not (yet) able to observe their faint/dwarf companion galaxies. Therefore we consider them either as relatively isolated galaxies, or as groups with only one bright galaxy within the observational window of magnitudes. We call them "the most isolated galaxies" instead of just "isolated galaxies" elsewhere in the paper. A detailed comparison of the galaxy luminosity functions of different populations (Tempel et al. 2009) suggests that the most isolated bright galaxies are the main galaxies of the groups with the remaining galaxies outside of the visibility window. As we mentioned, our main purpose in the SDSS group studies is to find the luminosity density field. For this, it is not important how any particular galaxy contributes to the large scale density (luminosity) field, either as a single galaxy or a member of a pair.

As we stressed earlier (Paper II), various catalogues of groups of galaxies have rather different properties, although group-finding methods may be similar. The resulting samples or catalogues may depend on the purposes of study and/or on particular search parameters applied. Fortunately, many samples of groups are homogeneous as stated by authors and tested by other investigators. Tovmassian \& Plionis (2009) studied the homogeneity of our SDSS DR5 groups (Paper II) and concluded that the sample is homogeneous with respect to virial properties. Nurmi et al. (in preparation) studied $N$-body simulations of dark matter halos comparing the properties of simulated halos with our volume-limited groups of galaxies. The results of this comparison show good agreement between our DR7 groups and their $N$-body simulations.

\subsection{Properties of groups}

The final results of group search have to be homogeneous samples of groups with physical properties important for studies of groups. The most important problem (not solved so far) is the dynamical status of groups of galaxies.

Recently Paz et al. (2006) and Plionis et al. (2006) studied shapes and virial properties of the 2dFGRS 2PIGG groups, the SDSS DR3 groups, and groups in numerical simulations, and found a strong dependence on richness.

Tovmassian \& Plionis (2009) studied the orientation and shape of the SDSS DR5 groups and found evidence that "groups of galaxies do not constitute a family of objects in dynamical equilibrium but rather are at various stages of their virialisation process".

One of the most important properties of groups of galaxies characterising their dynamical status is the existence of subgroups and -structures confirmed by many studies (Oegerle \& Hill 2001; Burgett et al. 2004; Coziol et al. 2009), Einasto et al. (in preparation). These studies also support the nonvirialised nature of groups of galaxies.

At the same time Berlind et al. (2006) studied the properties of groups, which were constructed so that each group corresponded to a single galaxy halo, and concluded that "most of their groups are likely virialised systems". With both observational and simulated data Niemi et al. (2007) showed that about $20 \%$ of nearby groups are not bound, but merely groups in a visual sense.

Controversial results obtained by various authors may be an indication that our present knowledge of groups of galaxies is poor. An optimistic viewpoint (supported by the authors of this paper) is that the study of a broad and inhomogeneous class of galaxy systems - groups of galaxies - will help step by step to solve important problems of galaxy formation and evolution, and of the large scale structure.

\subsection{Conclusions}

We have used the Sloan Digital Sky Survey Data Releases 7 to create flux- and volume-limited catalogues of groups of galaxies. Our main results are the following:

1. We present a flux-limited catalogue of groups of galaxies for the SDSS DR7. We applied the FoF method with a slightly increasing (empirically calibrated) linking length.

2. We created volume-limited samples of the DR7 groups of galaxies. We paid attention to the difficult problem of how to define a volume-limited sample of groups of galaxies, and discussed briefly some properties and applications of these groups.

3. We studied in detail selection effects caused by flux-limited galaxy samples. The two most important effects are the decrease of the group density and its richness with increasing distance from the observer. The growth of the mean size of groups is almost compensated by the absence of faint galaxies in the observed groups at large distances. The remaining bright galaxies form a compact core of the group. This confirms the similar luminosity-density relation found for the 2dFGRS groups earlier (Paper I).

4. We calibrated the properties of groups to empirically determine the scaling of the group properties and that of the FoF linking length with distance. As the SDSS main galaxy catalogue and the 2dFGRS galaxies have similar redshift distributions and luminosity functions, the linking length scaling laws for these catalogues are very close, but only up to the redshift $z=0.14$. Beyond this redshift the linking lengths for the SDSS groups decrease sharply. At higher redshifts we detect mainly compact cores of the groups due to a more narrow magnitude range (visibility window) of the SDSS. Thus the scaling law demonstrates the homogeneity limit of a group catalogue.

5. The properties of our groups confirm that selection effects have been properly taken into account when generating group catalogues.

Acknowledgements. Funding for the Sloan Digital Sky Survey (SDSS) and SDSS-II has been provided by the Alfred P. Sloan Foundation, the Participating Institutions, the National Science Foundation, the U.S. Department of Energy, the National Aeronautics and Space Administration, the Japanese Monbukagakusho, and the Max Planck Society, and the Higher Education Funding Council for England. The SDSS Web site is http://wWw . sdss.org/. The SDSS is managed by the Astrophysical Research Consortium (ARC) for the Participating Institutions. The Participating Institutions are the American 
Museum of Natural History, Astrophysical Institute Potsdam, University of Basel, University of Cambridge, Case Western Reserve University, The University of Chicago, Drexel University, Fermilab, the Institute for Advanced Study, the Japan Participation Group, The Johns Hopkins University, the Joint Institute for Nuclear Astrophysics, the Kavli Institute for Particle Astrophysics and Cosmology, the Korean Scientist Group, the Chinese Academy of Sciences (LAMOST), Los Alamos National Laboratory, the Max-Planck-Institute for Astronomy (MPIA), the Max-Planck-Institute for Astrophysics (MPA), New Mexico State University, Ohio State University, University of Pittsburgh, University of Portsmouth, Princeton University, the United States Naval Observatory, and the University of Washington.

We acknowledge the Estonian Science Foundation for support under grants No. 8005, 7765, and 7146, and the Estonian Ministry for Education and Science support by grant SF0060067s08. P.H. was supported by Societas Scientiarum Fennica, and Jenny and Antti Wihuri foundation. P.N. was supported by the Academy of Finland. We appreciate Volker Müller for fruitful discussions. We thank the referee for useful comments and corrections, which improved the paper.

\section{References}

Abazajian, K. N., Adelman-McCarthy, J. K., Agüeros, M. A., et al. 2009, ApJS, 182,543

Abell, G. O. 1958, ApJS, 3, 211

Abell, G. O. 1977, ApJ, 213, 327

Abell, G. O., Corwin, Jr., H. G., \& Olowin, R. P. 1989, ApJS, 70, 1

Adelman-McCarthy, J. K., Agüeros, M. A., Allam, S. S., et al. 2006, ApJS, 162, 38

Adelman-McCarthy, J. K., Agüeros, M. A., Allam, S. S., et al. 2007, ApJS, 172, 634

Adelman-McCarthy, J. K., Agüeros, M. A., Allam, S. S., et al. 2008, ApJS, 175, 297

Aguerri, J. A. L., Sánchez-Janssen, R., \& Muñoz-Tuñón, C. 2007, A\&A, 471, 17

Bahcall, N. A., McKay, T. A., Annis, J., et al. 2003, ApJS, 148, 243

Berlind, A. A., Frieman, J., Weinberg, D. H., et al. 2006, ApJS, 167, 1

Blanton, M. R., \& Roweis, S. 2007, AJ, 133, 734

Blanton, M. R., Brinkmann, J., Csabai, I., et al. 2003a, AJ, 125, 2348

Blanton, M. R., Hogg, D. W., Bahcall, N. A., et al. 2003b, ApJ, 592, 819

Burgett, W. S., Vick, M. M., Davis, D. S., et al. 2004, MNRAS, 352, 605

Christensen, C. G. 1975, AJ, 80, 282

Colless, M., Dalton, G., Maddox, S., et al. 2001, MNRAS, 328, 1039

Colless, M., Peterson, B. A., Jackson, C., et al. 2003,

[arXiv: astroph/0306581], technical report

Coziol, R., Andernach, H., Caretta, C. A., Alamo-Martínez, K. A., \& Tago, E. 2009, AJ, 137, 4795

De Propris, R., Couch, W. J., Colless, M., et al. 2002, MNRAS, 329, 87

Deng, X., He, J., Jiang, P., Luo, C., \& Wu, P. 2007, A\&A, 474, 783

Dong, F., Pierpaoli, E., Gunn, J. E., \& Wechsler, R. H. 2008, ApJ, 676, 868

Einasto, J., Saar, E., Kaasik, A., \& Chernin, A. D. 1974, Nature, 252, 111
Einasto, J., Klypin, A. A., Saar, E., \& Shandarin, S. F. 1984, MNRAS, 206, 529 Einasto, J., Einasto, M., Saar, E., et al. 2007a, A\&A, 462, 397

Einasto, J., Einasto, M., Tago, E., et al. 2007b, A\&A, 462, 811

Einasto, J., Hütsi, G., Einasto, M., et al. 2003a, A\&A, 405, 425

Einasto, J., Tago, E., Einasto, M., \& Saar, E. 2005, in Nearby Large-Scale Structures and the Zone of Avoidance, ed. A. P. Fairall, \& P. A. Woudt, ASP Conf. Ser., 329, 27

Einasto, M., Tago, E., Jaaniste, J., Einasto, J., \& Andernach, H. 1997, A\&AS, 123, 119

Einasto, M., Einasto, J., Müller, V., Heinämäki, P., \& Tucker, D. L. 2003b, A\&A, 401, 851

Einasto, M., Jaaniste, J., Einasto, J., et al. 2003c, A\&A, 405, 821

Eke, V. R., Baugh, C. M., Cole, S., et al. 2004, MNRAS, 348, 866

Goto, T. 2005, MNRAS, 359, 1415

Goto, T., Sekiguchi, M., Nichol, R. C., et al. 2002, AJ, 123, 1807

Harrison, E. R. 1974, ApJL, 191, L51+

Huchra, J. P., \& Geller, M. J. 1982, ApJ, 257, 423

Kiang, T. 1976, MNRAS, 174, 425

Kim, R. S. J., Kepner, J. V., Postman, M., et al. 2002, AJ, 123, 20

Koester, B. P., McKay, T. A., Annis, J., et al. 2007, ApJ, 660, 239

Lee, B. C., Allam, S. S., Tucker, D. L., et al. 2004, AJ, 127, 1811

Martínez, V. J., \& Saar, E. 2002, Statistics of the Galaxy Distribution (Boca

Raton: Chapman \& Hall/CRC)

Merchán, M., \& Zandivarez, A. 2002, MNRAS, 335, 216

Merchán, M. E., \& Zandivarez, A. 2005, ApJ, 630, 759

Miller, C. J., Nichol, R. C., Reichart, D., et al. 2005, AJ, 130, 968

Mottmann, J., \& Abell, G. O. 1977, ApJ, 218, 53

Nichol, R. C. 2004, in Clusters of Galaxies: Probes of Cosmological Structure and Galaxy Evolution, ed. J. S. Mulchaey, A. Dressler, \& A. Oemler, 24

Niemi, S., Nurmi, P., Heinämäki, P., \& Valtonen, M. 2007, MNRAS, 382, 1864 Oegerle, W. R., \& Hill, J. M. 2001, AJ, 122, 2858

Paz, D. J., Lambas, D. G., Padilla, N., \& Merchán, M. 2006, MNRAS, 366, 1503 Plionis, M., Basilakos, S., \& Ragone-Figueroa, C. 2006, ApJ, 650, 770

Popesso, P., Biviano, A., Böhringer, H., \& Romaniello, M. 2007, A\&A, 464, 451 Press, W. H., \& Davis, M. 1982, ApJ, 259, 449

Tago, E., Einasto, J., Saar, E., et al. 2006, Astron. Nachr., 327, 365

Tago, E., Einasto, J., Saar, E., et al. 2008, A\&A, 479, 927

Tempel, E., Einasto, J., Einasto, M., Saar, E., \& Tago, E. 2009, A\&A, 495, 37

Tovmassian, H. M., \& Plionis, M. 2009, ApJ, 696, 1441

Tucker, D. L., Oemler, A. J., Hashimoto, Y., et al. 2000, ApJS, 130, 237

Turner, E. L., \& Gott, III, J. R. 1976, ApJS, 32, 409

Weinmann, S. M., van den Bosch, F. C., Yang, X., \& Mo, H. J. 2006, MNRAS, 366, 2

Wen, Z. L., Han, J. L., \& Liu, F. S. 2009, ApJS, 183, 197

Yang, X., Mo, H. J., van den Bosch, F. C., \& Jing, Y. P. 2005, MNRAS, 356, 1293

Yang, X., Mo, H. J., van den Bosch, F. C., et al. 2007, ApJ, 671, 153

Yoon, J. H., Schawinski, K., Sheen, Y., Ree, C. H., \& Yi, S. K. 2008, ApJS, 176, 414

Zandivarez, A., Martínez, H. J., \& Merchán, M. E. 2006, ApJ, 650, 137

Zeldovich, I. B., Einasto, J., \& Shandarin, S. F. 1982, Nature, 300, 407 